\title{
The exopolysaccharide-eDNA interaction modulates 3D architecture of Bacillus subtilis biofilm
}

\author{
Na Peng ${ }^{1}$, Peng Cai ${ }^{1 *}$, Monika Mortimer ${ }^{2}$, Yichao $\mathrm{Wu}^{1}$, Chunhui Gao $^{1}$ and Qiaoyun Huang ${ }^{1}$
}

\begin{abstract}
Background: Bacterial biofilms are surface-adherent microbial communities in which individual cells are surrounded by a self-produced extracellular matrix of polysaccharides, extracellular DNA (eDNA) and proteins. Interactions among matrix components within biofilms are responsible for creating an adaptable structure during biofilm development. However, it is unclear how the interactions among matrix components contribute to the construction of the three-dimensional (3D) biofilm architecture.

Results: DNase I treatment significantly inhibited Bacillus subtilis biofilm formation in the early phases of biofilm development. Confocal laser scanning microscopy (CLSM) and image analysis revealed that eDNA was cooperative with exopolysaccharide (EPS) in the early stages of B. subtilis biofilm development, while EPS played a major structural role in the later stages. In addition, deletion of the EPS production gene epsG in B. subtilis SBE1 resulted in loss of the interaction between EPS and eDNA and reduced the biofilm biomass in pellicles at the air-liquid interface. The physical interaction between these two essential biofilm matrix components was confirmed by isothermal titration calorimetry (ITC).

Conclusions: Biofilm 3D structures become interconnected through surrounding eDNA and EPS. eDNA interacts with EPS in the early phases of biofilm development, while EPS mainly participates in the maturation of biofilms. The findings of this study provide a better understanding of the role of the interaction between eDNA and EPS in shaping the biofilm 3D matrix structure and biofilm formation.
\end{abstract}

Keywords: Extracellular DNA (eDNA), Exopolysaccharide (EPS), Bacillus subtilis, Biofilm formation

\section{Background}

Bacterial biofilms are heterogeneous communities that exhibit a remarkable degree of spatiotemporal organization [1-3]. The spatial architecture of multicellular communities depends on the production of extracellular matrix, which is mainly composed of polysaccharides, proteins, and extracellular DNA (eDNA) $[4,5]$. Extracellular DNA, as an important matrix component in biofilms [5, 6], can be used by bacteria for several vital functions; for example, as

\footnotetext{
* Correspondence: cp@mail.hzau.edu.cn

'State Key Laboratory of Agricultural Microbiology, College of Resources of Environment, Huazhong Agricultural University, Wuhan 430070, China Full list of author information is available at the end of the article
}

structural components of biofilms [7], nutrient sources [8], and a gene pool for horizontal gene transfer (HGT) [9]. The significance of eDNA in biofilm formation has been studied in Pseudomonas aeruginosa [6], Staphylococcus epidermidis [10], Streptococcus pneumoniae [11] and Vibrio cholerae [12]. In these studies, young biofilms were easily disturbed by DNase I treatment, but this treatment was not effective against aged biofilms. This loss of sensitivity to DNase I treatment suggests that in mature biofilms, either other extracellular matrix components complement or replace eDNA functions or that eDNA is shielded from enzymatic degradation when bound to other biofilm components. 
Exopolysaccharide (EPS) is one of the major extracellular biofilm matrixes [13-15]. Interactions between EPS and eDNA have been investigated in some bacterial biofilms. In Streptococcus mutans biofilms, the interaction between eDNA and glucan results in the formation of filamentous structures that play an important role in connecting bacterial cells [16]. In the case of $P$. aeruginosa, eDNA and the exopolysaccharide Psl physically interact in biofilms to form the web of Psl-eDNA fibres, which function as a skeleton facilitating bacterial adhesion and growth [17]. Meanwhile, Psl can interact with genomic DNA from human neutrophils or strains of $S$. aureus, implying that $P$. aeruginosa can utilize genomic DNA from other organisms to form its own community [17]. Therefore, the eDNA-EPS interaction is important for the construction of biofilm architecture.

Bacillus subtilis, a gram-positive bacterium, produces a variety of biologically active compounds with a broad spectrum of activities against plant pathogens [18-23]. Due to the role of $B$. subtilis as a biocontrol agent in agricultural settings, a growing number of studies have focused on biofilm formation under natural and artificial conditions [18, 24-26]. Exopolysaccharide (EPS) is a key component in the B. subtilis matrix that promotes cell binding in structural biofilms [27]. It has been proposed that eDNA released by dead cells during the process of cannibalism in B. subtilis 168 could be related to matrix development [28-31]. On the other hand, eDNA released from B. subtilis 3610 during the stationary phase is not involved in biofilm establishment [32]. However, how interactions between eDNA and EPS modulate $B$. subtilis biofilm formation processes and architecture construction is less known.

This study focused on elucidating (1) the role of eDNA in the construction of the $B$. subtilis biofilm threedimensional (3D) architecture and (2) the interaction between eDNA and EPS during biofilm formation. Here, we used confocal laser scanning microscopy (CLSM) and image analysis to investigate the role of eDNA during $B$. subtilis SBE1 biofilm structure formation. The $\triangle$ eps $G$ strain (deletion of one EPS production gene) was used to examine the role of EPS and its interaction with eDNA during biofilm development. To better understand the interactions of EPS and eDNA, isothermal titration calorimetry (ITC) was used to study the thermodynamics of the interactions between these two molecules.

\section{Results}

The role of extracellular DNA (eDNA) in the construction of the B. subtilis SBE1 biofilm three-dimensional (3D) architecture

In order to understand the contribution of eDNA in the biofilm formation of $B$. subtilis SBE1, the impact of DNase I on biofilm formation was tested using a static biofilm assay. The formation of biofilms grown for 3, 6, and $12 \mathrm{~h}$ with DNase I was clearly suppressed compared with the untreated control, based on crystal violet assay (3 h, $P=0.0020 ; 6$ h, $P=0.0003 ; 12$ h, $P=0.0000$ ) (Fig. 1a). In contrast, biofilms grown with DNase I for 24 and $48 \mathrm{~h}$ were not significantly different from biofilms grown without DNase I (Fig. 1a). Furthermore, as shown in Fig. $1 \mathrm{~b}$, the biofilms that were 3,6 , and $12 \mathrm{~h}$ old when the DNase I treatments were initiated were dissolved $(3 \mathrm{~h}$, $P=0.420 ; 6 \mathrm{~h}, P=0.0392 ; 12 \mathrm{~h}, P=0.0005)$, whereas the biofilms that were 24 and $48 \mathrm{~h}$ old at the time of DNase I exposure were only affected to a minor degree. The biofilm treated with DNase I after $12 \mathrm{~h}$ was further characterized using atomic force microscopy (AFM) to measure the depth of the furrows generated (Fig. 2). In the absence of DNase I, furrows between cells were $\sim 200$ $\mathrm{nm}$ in depth (Fig. 2a, b and e). In the presence of DNase I, the furrows of the expanding biofilm were significantly
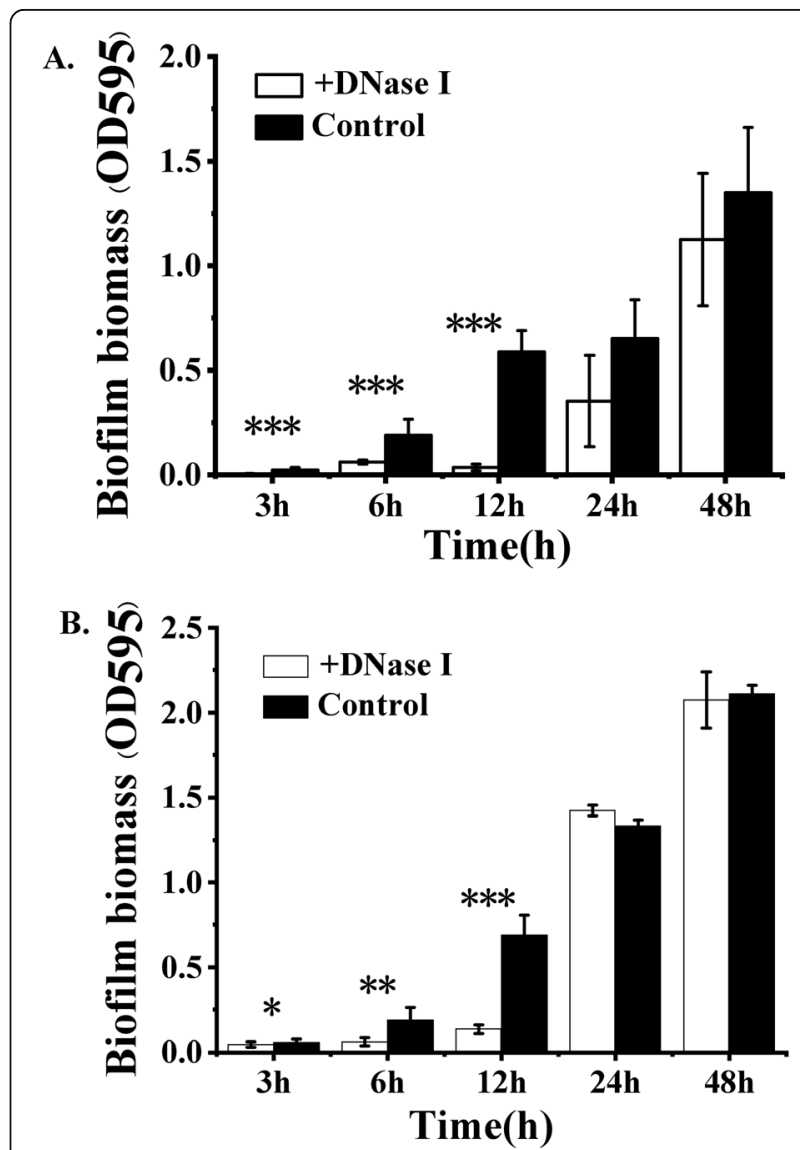

Fig. 1 Effect of extracellular DNA (eDNA) removal on biofilm formation in microtiter plates. DNase I was either added at the beginning of the experiment (a) or after the biofilm had established. b. Biomass was quantified by using a crystal violet assay. White bars = DNase I treated, black bars = untreated control. The bars are means of five replicates, and the error bars represent standard deviations. ${ }^{*} P<0.05,{ }^{* *} P<0.01,{ }^{* * *} P<0.001$ for comparisons of data obtained in the absence of DNase I and in the presence of DNase I 
deeper (up to $400 \mathrm{~nm}$ ) than those formed in the absence of DNase I (Fig. 2c, d and e) $(P<0.001)$, suggesting that the gaps between cells in biofilms may be filled with eDNA. Therefore, eDNA may be an adhesion compound enabling cell-to-cell attachment, which initiates biofilm formation.

To further understand how eDNA functions as a cellcell adhesin during biofilm development, the construction of an eDNA matrix in a biofilm was examined over time. The formation and sequence of assembly of eDNA changed over time. After $12 \mathrm{~h}$ of biofilm development (Fig. 3a), the cells were densely packed in the eDNA matrix, forming a 3D biofilm structure (termed the eDNA-microcolony complex, highlighted with red box). After $24 \mathrm{~h}$, the biofilm structures expanded in several dimensions with less eDNA-matrix enmeshed in and around the bacteria (Fig. 3b and c). Imaris analysis also showed that the content of eDNA in the biofilms was substantially reduced after $24 \mathrm{~h}$ (Fig. $4 \mathrm{~b}$ ). These results suggested that other matrix components (i.e., exopolysaccharides (EPS)) may complement eDNA as cell-cell adhesins when the biofilm becomes mature or perhaps that eDNA in mature biofilms interacts with other biomolecules that can protect the eDNA from DNase degradation. As shown in Fig. S1, in the following $24 \mathrm{~h}$, more bacteria gathered in the biofilms. Meanwhile, the amount of eDNA increased again in the 48-h biofilm (Fig. 4), suggesting that eDNA may assist other matrix components during biofilm maturation.

\section{Exopolysaccharide (EPS) and eDNA colocalization in $B$. subtilis SBE1 3D biofilms}

Exopolysaccharide (EPS) is an important extracellular biofilm matrix in B. subtilis. The spatial assembly of EPS and eDNA at each developmental stage of wild-type biofilms was examined at 12,24 and $48 \mathrm{~h}$ by confocal laser scanning microscopy (CLSM) (Fig. 4). Figure 4Aa shows the $3 \mathrm{D}$ evolution of the EPS-eDNA interaction over time. The cross-sectional images at each time point are shown in Fig. 4Ab-j. At the 12-h time point, the biofilms contained considerable eDNA, which connected bacteria (Fig. 4A a (1)); at the 24-h time point, most eDNA was peripherally localized, and EPS was found to concentrate inside the biofilms (Fig. 4Aa (2)); at the 48-h time point, eDNA and EPS covered the entire structure (Fig. 4Aa (3)).

This observation was supported by the quantitative colocalization analysis. As shown in Fig. 5, Pearson's coefficient (PC) defines the quality of a linear relationship between two signals. Mander's coefficients are based on Pearson's correlation coefficient, and the average intensity values are obtained from the mathematical expression. The thresholded Mander's coefficients were calculated by setting the threshold to the estimated value of background instead of zero. The analysis of seven image stacks by these methods showed the complete colocalization of
eDNA-EPS in wild-type biofilms (Fig. 5a). The thresholded Mander's $\mathrm{tM}_{1}$ (M1thr) indicated the fraction of eDNA (TOTO-1 signal, green) overlapping with EPS (ConA signal, orange), and $\mathrm{tM}_{2}$ (M2thr) indicated the fraction of EPS overlapping eDNA. The PC (black bar) will tend to 0 when random noise is added to complete colocalizing structures. At $12 \mathrm{~h}$, a wider distribution of eDNA was observed in the intercellular space compared to EPS. Most of the EPS overlapped with the eDNA (M1thr $<0.5$, M2thr $>0.5$ ). At $24 \mathrm{~h}$, most of the eDNA overlapped with the EPS (M1thr $>0.5$, M2thr $<0.5)$. At 48 $\mathrm{h}$, the eDNA and EPS were completely colocalized (M1thr $>0.5$, M2thr $>0.5)$. This quantitative analysis was consistent with the CLSM observations, suggesting that eDNA was cooperative with EPS in early stages, while EPS might play a larger role in the later stages of $B$. subtilis SBE1 biofilm development.

\section{Spatial assembly of EPS and eDNA in biofilms of the $B$. subtilis SBE1 epsG mutant}

To further confirm the spatial assembly of EPS and eDNA during the development of B. subtilis SBE1 biofilms, an EPS defective mutant was constructed. The genome of $B$. subtilis SBE1 contains homologous epsA-O genes (the genetic locus for the eps $G$ gene is as follows: open reading frame Query_1049240 [epsG]). As shown in Fig. S2, the $\Delta e p s G$ mutant produced significantly less cell-associated EPS than wild-type cells. The deletion of the EPS production gene epsG in B. subtilis SBE1 also resulted in a reduction in biofilm biomass in pellicles at the air-liquid interface (Fig. S1). Then, the spatial assembly of EPS and eDNA at each developmental stage of $\Delta$ eps $G$ mutant biofilms was also examined at 12,24 and $48 \mathrm{~h}$ by CLSM (Fig. 6). Quantitative image analysis showed that there was a substantial reduction in the contents of both eDNA and EPS in the biofilms of the AepsG mutant compared to wild-type B. subtilis SBE1 (Figs. 4 and 6). And quantitative colocalization analysis showed that eDNA colocalized with EPS in B. subtilis SBE1 pellicles but not in $\triangle e p s G$ strain pellicles. In addition, the eDNA and EPS in $\triangle$ eps $G$ mutant biofilms exhibited partial colocalization (12 h, 24h) (M1thr $<0.5$, M2thr <0.5) (Fig. 5b). These results confirmed that eDNA interacts with EPS during biofilm development.

The colocalization of eDNA and EPS in B. subtilis SBE1 pellicles suggested the potential physical interaction between these two components. Isothermal titration calorimetry (ITC) is an important technique to study the thermodynamics of molecular interactions. The interaction between DNA and EPS could be determined by entropy changes $(\Delta H)$ because of the binding between them. Consistently, isothermal titration calorimetry results showed that the total heat produced from DNA from B. subtilis SBE1, B. subtilis 3610, Shewanella 


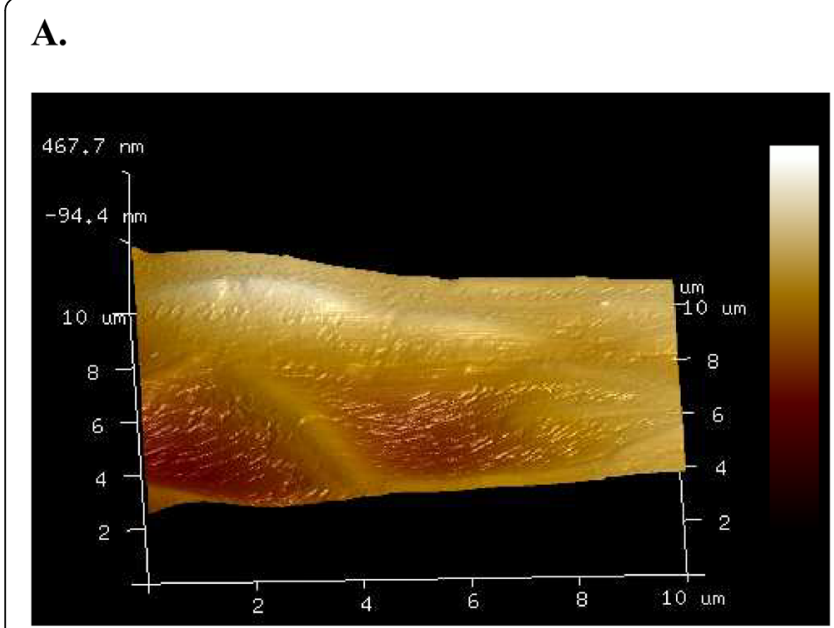

C.

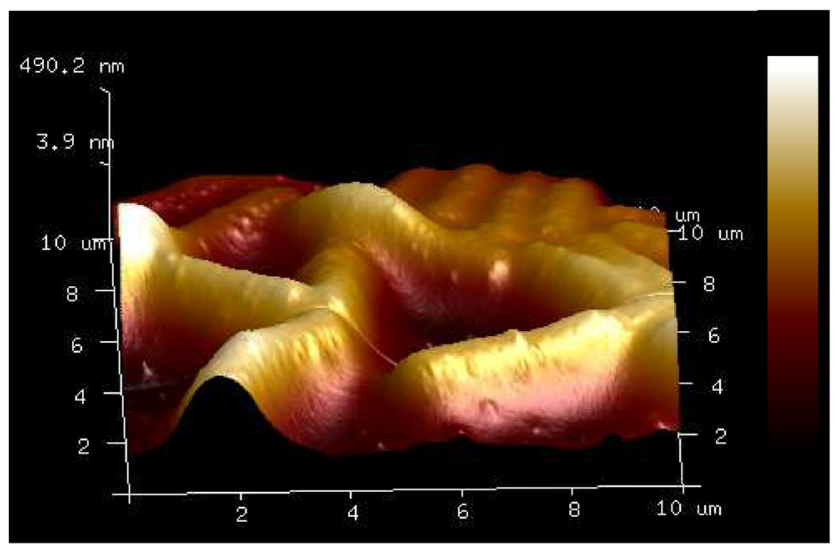

E.

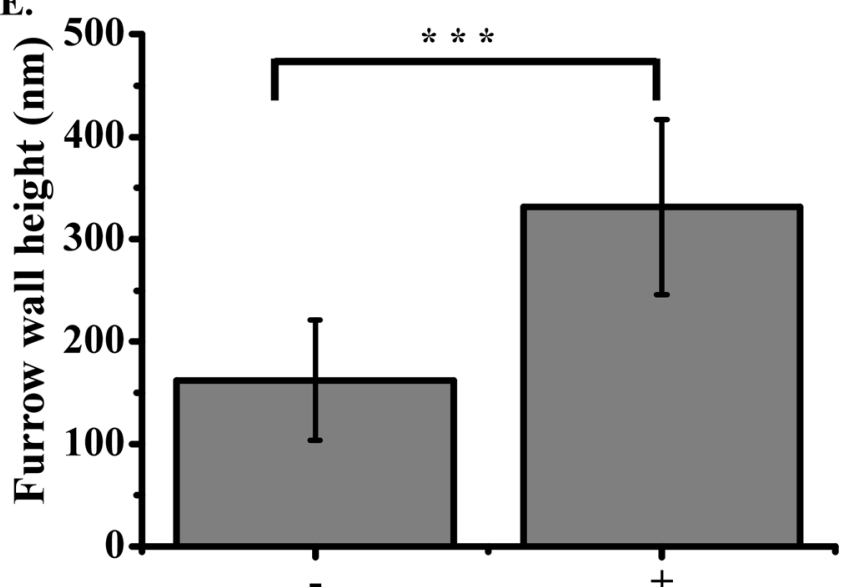

B.

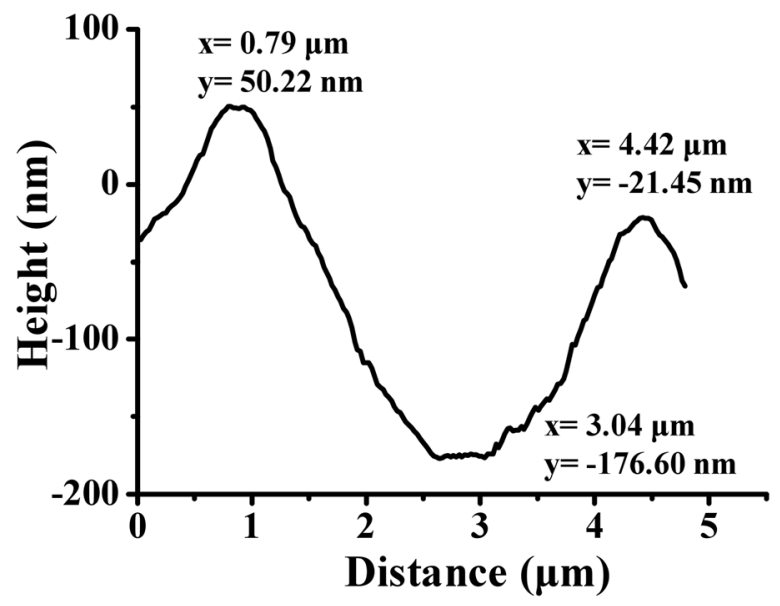

D.

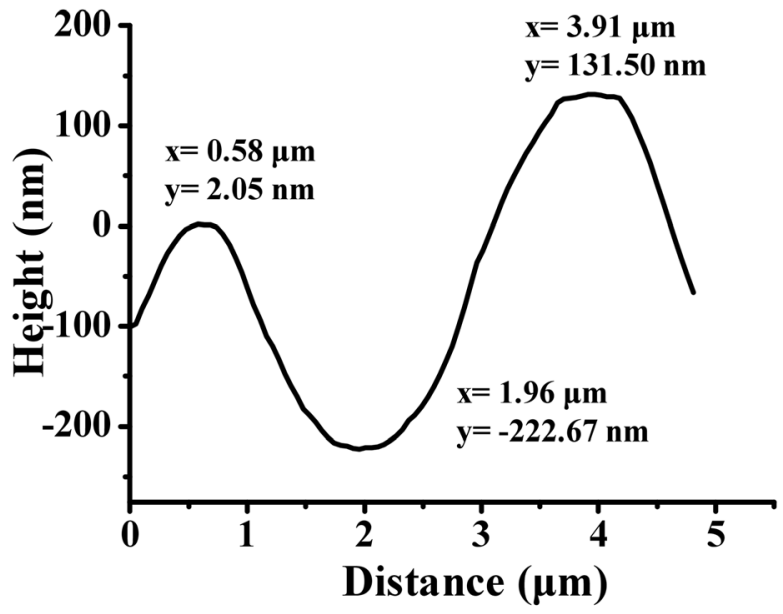

Fig. 2 Atomic force microscopy (AFM) surface profiles of biofilms (12 h). 3D AFM images of biofilms cultured in the absence (a) and presence of DNase I (c). Scale is a relative color scale. A is scaled to $467.7 \mathrm{~nm}$, and C is scaled to $490.2 \mathrm{~nm}$. Measurements were taken between cells to generate a depth profile as shown in $\mathbf{b}$ and $\mathbf{d}$. The $y$-axis scale is different for $\mathbf{b}$ and $\mathbf{d}$. e Depths of furrows between cells from biofilms cultured in the absence $(-, n=10$ from three AFM images) and presence $(+, n=10$ from three AFM images) of DNase I. Error bars represent standard deviations. ${ }^{* * *} P<0.001$ 

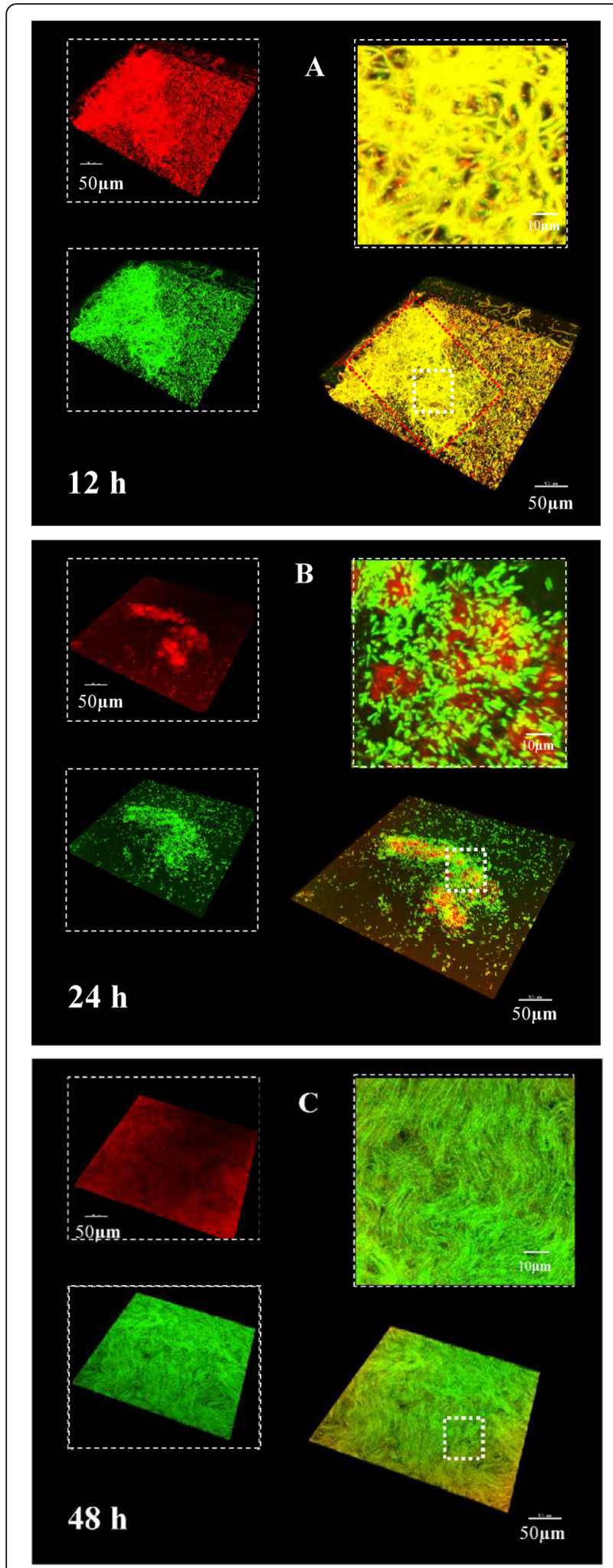

Fig. 3 Dynamics of morphogenesis, three- dimensional (3D) architecture development and microbial population shifts of $B$. subtilis SBE1 biofilms. The biofilms stained with TOTO-1 for eDNA (green) and SYTO 60 for bacteria (red). Representative 3D rendering images of $B$. subtilis SBE1 biofilms at $12 \mathrm{~h} \mathrm{(a),} 24 \mathrm{~h} \mathrm{(b)}$ and $48 \mathrm{~h}$ (c). At the upper left of each panel, the two channels are displayed separately, while the merged image is displayed at the bottom right. A magnified (close-up) view of each small box depicted in the merged image is positioned in the upper right corner of each panel

oneidensis MR1 and P. putida KT2440 binding to EPS was $(1.5 \pm 0.2) \times 10^{-4}, \quad(2.4 \pm 0.3) \times 10^{-4}, \quad(9.5 \pm 0.5) \times$ $10^{-4}$ and $(8.6 \pm 0.4) \times 10^{-4} \mathrm{KJ}(n=3)$, respectively (Fig. S3E). The calculated binding enthalpy (the binding heat per DNA molecule) of EPS was $222.72 \pm 26.34,162.77 \pm$ $17.82,338.46 \pm 45.23$ and $398.50 \pm 38.67 \mathrm{~kJ} / \mathrm{mol}(n=3)$ for B. subtilis SBE1, B. subtilis 3610, Shewanella oneidensis MR1 and P. putida KT2440, respectively (Fig. S3F), indicating that the interaction between DNA from MR1 and KT2440 and EPS is more exothermic than DNA from SBE1 and 3610. However, EPS of B. subtilis SBE1 can interact with DNA from these bacteria, which might enable $B$. subtilis SBE1 cells to bind eDNA from these bacteria, leading to biofilm formation.

\section{Discussion}

Interactions among matrix components within biofilms are responsible for creating an adaptable structure during biofilm development. However, how interactions between extracellular DNA (eDNA) and exopolysaccharide (EPS) modulate B. subtilis biofilm formation processes and architecture construction is less known. In this study, we focused on elucidating (1) the role of eDNA in the construction of the $B$. subtilis biofilm threedimensional (3D) architecture and (2) the interaction between eDNA and EPS during biofilm formation.

We found that B. subtilis SBE1 biofilms were dissolved when the DNase I treatments were initiated, whereas the biofilms after $24 \mathrm{~h}$ at the time of DNase I exposure were only affected to a minor degree. Young biofilms are easily removed by DNase, but DNase treatment is not effective once the biofilm has aged past a certain point. Such a transition has been documented for, for example, S. epidermidis (at $12 \mathrm{~h}$ ) [10], P. aeruginosa (at $80 \mathrm{~h}$ ) [6], and Vibrio cholera (at $72 \mathrm{~h}$ ) [12]. The use of DNase for biofilm removal is effective but dependent on the age of the biofilm. What causes the resistance of the biofilm to DNase remains to be explored, but this temporary sensitivity suggests either that other extracellular matrix components replace or complement eDNA within the mature biofilm or that eDNA is bound by another component that shields it from enzymatic degradation. Similar results have been observed in Listeria monocytogenes that peptidoglycan, as an additional essential component, 


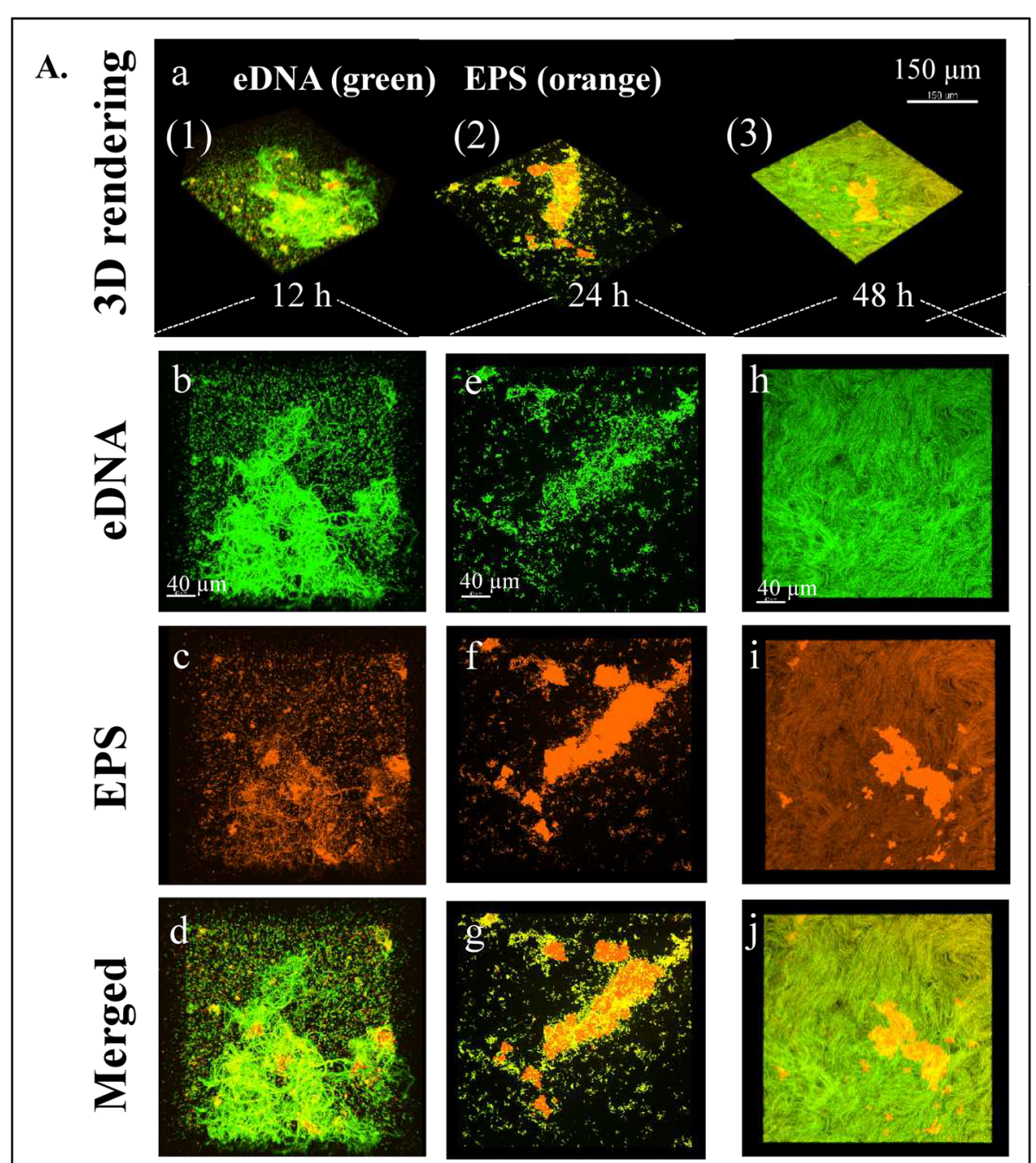

B.

\begin{tabular}{cccc}
\hline Biomass $\left(\boldsymbol{\mu \mathbf { m } ^ { 3 } / \boldsymbol { \mu m } ^ { 2 } )}\right.$ & $\mathbf{1 2} \mathbf{h}$ & $\mathbf{2 4} \mathbf{h}$ & $\mathbf{4 8 ~ h}$ \\
\hline eDNA & $1.29 \pm 0.21$ & $0.89 \pm 0.26$ & $1.96 \pm 0.14$ \\
EPS & $1.09 \pm 0.12$ & $1.80 \pm 0.22$ & $1.65 \pm 0.49$ \\
\hline
\end{tabular}

Fig. 4 Structural arrangement between exopolysaccharide (EPS) and extracellular DNA (eDNA) during biofilm development of B. subtilis SBE1 and its EPS mutant (LepsG). A Representative 3D renderings of B. subtilis SBE1 biofilms at 12, 24 and 48 h: (a) shows the dynamic evolution of biofilms over time. Panel (b-J) show cross sectional images of selected area for close-up views of structural organization of EPS (orange) and eDNA (green) during the development of biofilm matrix complex. B The biomass values of EPS and eDNA in the biofilms were calculated using Imaris. The data shown are mean values \pm SD $(n=3)$

is required for DNA-dependent biofilm development [33]. eDNA has been shown to play an important role in cell-to-cell interconnection during early $B$. subtilis SBE1 biofilm formation. It has been previously reported that cells can interact during the biofilm accumulation phase of $S$. aureus through recycled cytoplasmic proteins, which can be linked by eDNA [34]. Thus, another biofilm component may interact with eDNA to stabilize biofilm structure. 


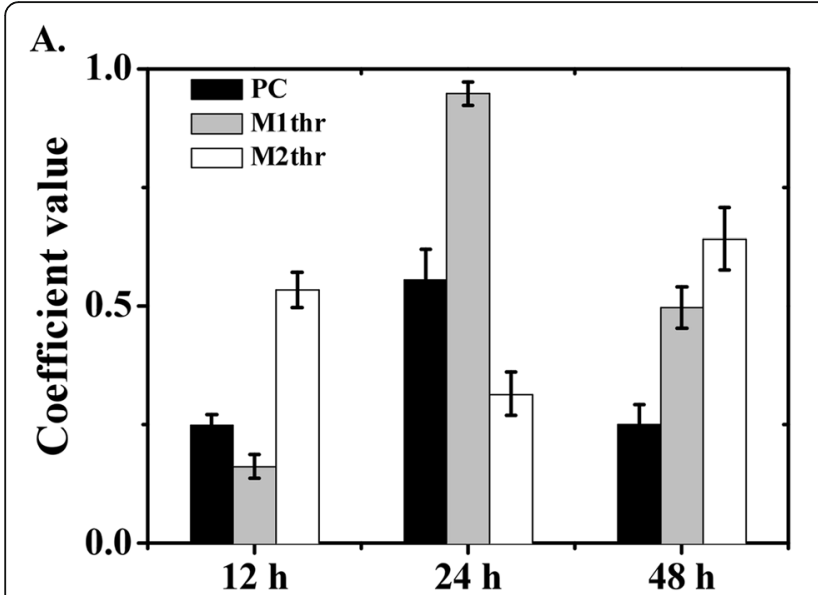

B.

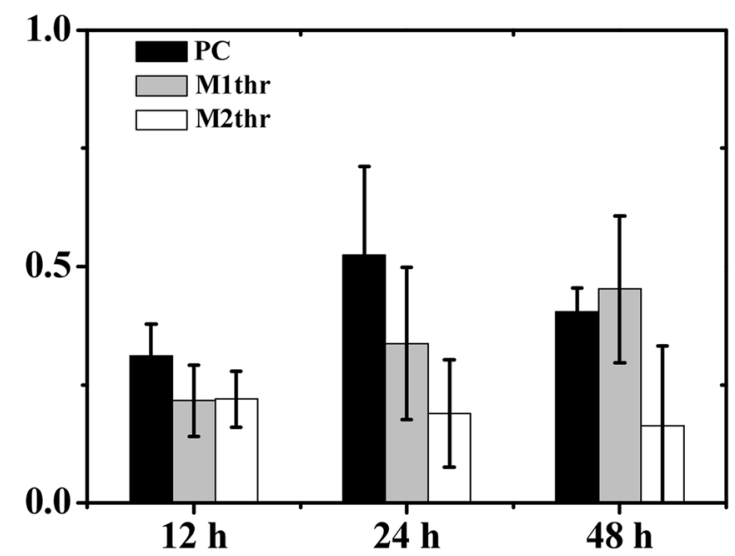

Fig. $\mathbf{5}$ The analysis of extracellular DNA (eDNA)-exopolysaccharide (EPS) colocalization coefficients in B. subtilis SBE1 (a) and its EPS mutant ( $\triangle e p s G$ ) (b) biofilms. Columns showed the eDNA-EPS colocalization coefficients analyzed from seven images by three methods: Pearson's correlation coefficient $(\mathrm{PC})$, the thresholded Mander's $\mathrm{tM}_{1}$ (M1thr) representing fraction of eDNA overlapping EPS, and $\mathrm{tM}_{2}$ (M2thr) representing fraction of EPS overlapping eDNA

Exopolysaccharide is an important extracellular biofilm matrix in B. subtilis. The colocalization of eDNA and EPS observed in native extracellular matrix provided evidence for direct interactions between eDNA and EPS in B. subtilis SBE1 biofilms. Previous studies have been reported that the major EPS component of all B. subtilis biofilms is synthesized by the products of the 15-gene operon eps $A-O$ (referred to as the eps operon) [27, 3538]. The molecular structure of EPS has yet to be elucidated. To date, only a subset of EPS genes has been studied individually. EpsA and EpsB act as tyrosine kinase modulators and tyrosine kinases, respectively, and both are required for biofilm formation [37]. EpsE is a bifunctional protein that coordinates the production of EPS with the cessation of motility [38]. EpsG is a protein that is presumably involved in EPS polymerization [27]. Among these genes, the deletion of eps $\mathrm{G}$ could prevent surface-adhered biofilm formation even in the $\Delta$ sip W suppressor strain [39]. The above-described results indicate that eDNA may cooperate with EPS, which promotes cell-cell adhesion during early biofilm development. To confirm this, the $\Delta$ eps $G$ mutant was constructed to weaken the function of surface adhesion of EPS during biofilm formation. eDNA colocalized with EPS in B. subtilis SBE1 pellicles but not in $\triangle$ eps $G$ strain pellicles. There was also a substantial reduction in the contents of both eDNA and EPS in the biofilms of the $\triangle$ epsG mutant compared to wild-type B. subtilis SBE1. Biofilms formed by the $\triangle e p s \mathrm{G}$ mutant contained eDNA that did not colocalize with EPS in the biofilms. A similar pattern has been observed in P. aeruginosa PAO1. The Pel and Psl polysaccharides contribute to eDNA release and distribution during PAO1 biofilm development. Biofilms formed by the PAO1 1 pelA mutant contained eDNA in the inner parts of microcolony structures. Biofilms formed by the $\mathrm{PAO} 1 \Delta p s l \mathrm{BCD}$ mutant contained a small amount of eDNA close to the substratum of biofilms [40]. It is possible that epsG may be involved in eDNA release and distribution during B. subtilis SBE1 biofilm formation.

Extracellular DNA interacts with EPS in the early phases of biofilm development, while EPS played a major structural role in the later stages. This transition of the role of eDNA from initial construction of the 3D extracellular matrix to matrix microaggregation is similar to the role of eDNA and lipoteichoic acid (LTA) in biofilms of Streptococcus mutants [41]. The colocalization of eDNA and EPS in B. subtilis SBE1 pellicles suggested the potential physical interaction between these two components. Previous work has used Isothermal titration calorimetry (ITC) to study the molecular interaction between protein and lipid polysaccharide (LPS) [42]. The interaction between DNA from $S$. oneidensis MR and $P$. putida KT2440 and EPS is more exothermic than DNA from B. subtilis SBE1 and B. subtilis 3610. This selectivity between DNA and EPS may be driven by a small energy difference of the interactions, such as electrostatic and van der Waals forces [43]. However, EPS of B. subtilis SBE1 can interact with DNA from $S$. oneidensis and Pseudomonas putida which also inhabit the soil. These species that commonly share soil ecosystems with $B$. subtilis, maybe associated in multispecies biofilms. In the bulk soil, bacteria are found in patches or microcolonies containing low cell numbers, often composed of different bacterial species [44, 45]. When exposed to nutrient sources, these microcommunities have the potential to develop into multispecies biofilms with high bacterial density [45]. The first step of the succession in an early multispecies biofilm 


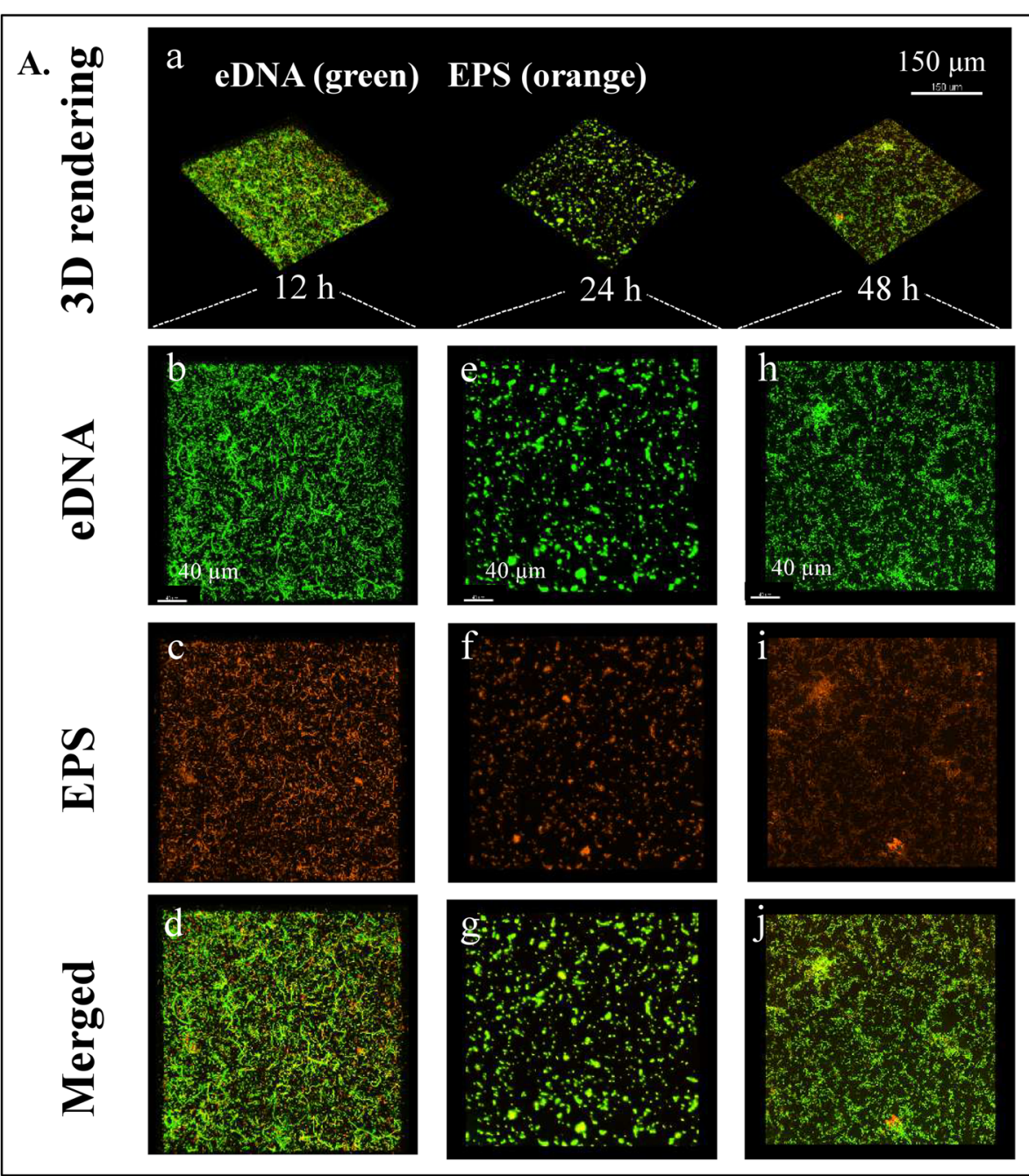

B.

\begin{tabular}{cccc}
\hline Biomass $\left(\boldsymbol{\mu \mathbf { m } ^ { 3 } / \boldsymbol { \mu m } ^ { 2 } )}\right.$ & $\mathbf{1 2} \mathbf{h}$ & $\mathbf{2 4} \mathbf{h}$ & $\mathbf{4 8 ~ h}$ \\
\hline eDNA & $0.73 \pm 0.11$ & $0.75 \pm 0.12$ & $0.77 \pm 0.03$ \\
& & & \\
EPS & $0.74 \pm 0.03$ & $0.81 \pm 0.14$ & $0.76 \pm 0.06$ \\
\hline
\end{tabular}

Fig. 6 Structural arrangement between exopolysaccharide (EPS) and extracellular DNA (eDNA) during biofilm development of EPS mutant (LepsG). A Representative 3D renderings of B. subtilis SBE1 biofilms at 12, 24 and $48 \mathrm{~h}$ : (a) shows the dynamic evolution of biofilms over time. Panel (b-J) show cross sectional images of selected area for close-up views of structural organization of EPS (orange) and eDNA (green) during the development of biofilm matrix complex. B The biomass values of EPS and eDNA in the biofilms were calculated using Imaris. The data shown are mean values $\pm \mathrm{SD}(n=3)$

based on the ability of surface/cell-cell attachment of soil bacteria [46]. Thus, EPS of B. subtilis can interact with DNA from these bacteria, which might enable $B$. subtilis cells to bind eDNA from these bacteria, initiating the biofilm formation.

\section{Conclusions}

Extracellular DNA (eDNA) and exopolysaccharide (EPS), two essential matrix components of the B. subtilis SBE1 biofilm, cooperate by physically interacting in bacterial biofilms. Over time, the biofilm three-dimensional (3D) 


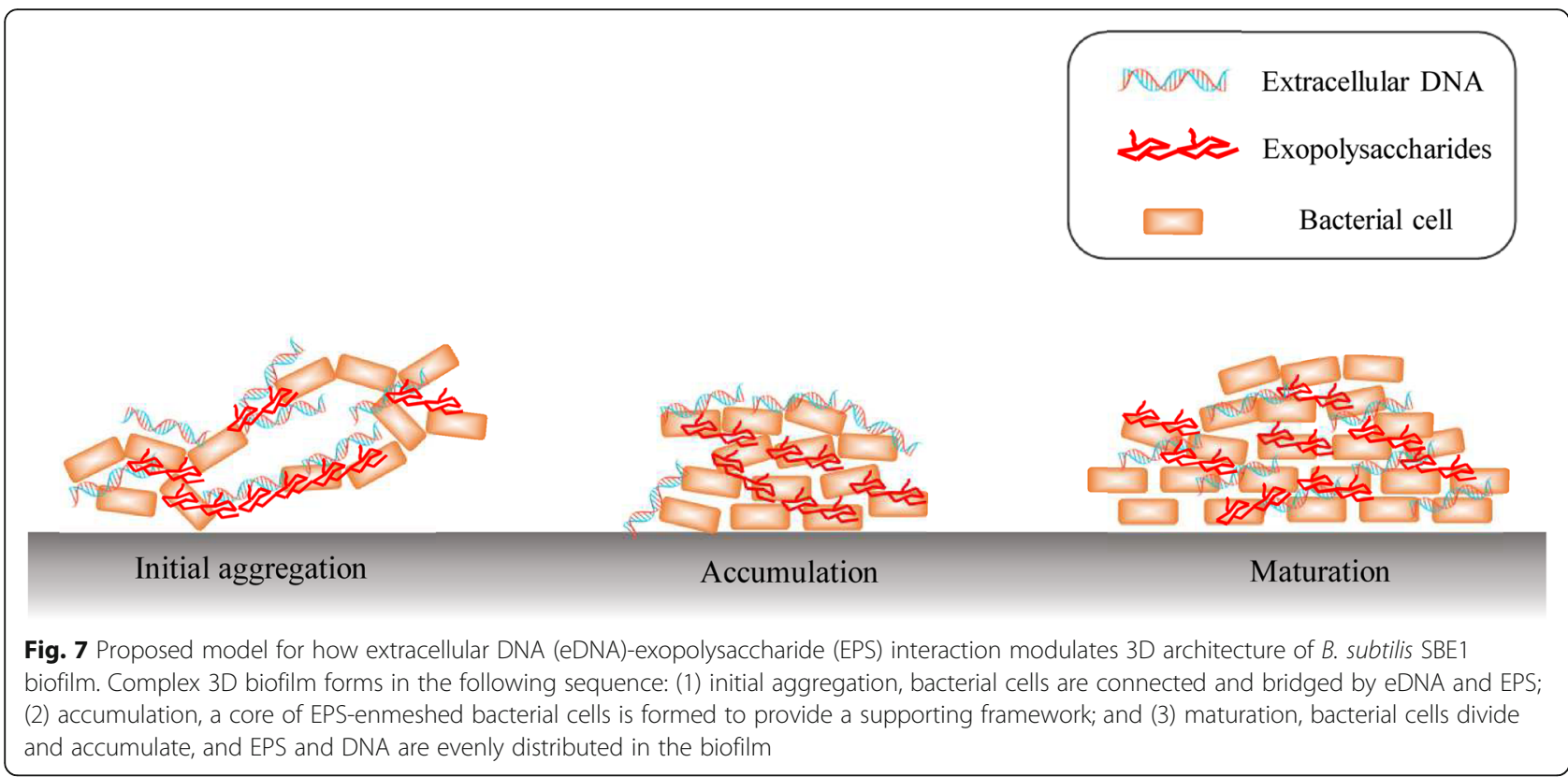

structures become interconnected through surrounding eDNA and EPS. eDNA interacts with EPS in the early phases of biofilm development, while EPS mainly participates in the maturation of biofilms. Based on our research, we proposed a model to describe how the eDNA-EPS interaction mediates the construction of the complex 3D biofilm architecture and establishes spatial heterogeneities in B. subtilis SBE1. Complex 3D biofilms form in the following sequence: (1) initial aggregation, bacterial cells are connected and bridged by eDNA and EPS; (2) accumulation, a core of EPS-enmeshed bacterial cells is formed to provide a supporting framework; and (3) maturation, bacterial cells divide and accumulate, and EPS and DNA are evenly distributed in the biofilm (Fig. 7). The interaction between eDNA and EPS plays a vital role in the construction of 3D biofilm architecture. In addition, the eDNA-EPS interaction might increase the survival of B. subtilis SBE1 in different environments by allowing eDNA from other microbial species to act as a scaffold on which a community can grow.

\section{Methods}

\section{Bacteria strains and cultivation conditions}

Bacillus subtilis SBE1, a wild-type soil isolate, was obtained from the State Key Laboratory of Agricultural Microbiology, Huazhong Agriculture University (Wuhan, China). B. subtilis SBE1 has been studied in our previous work where we showed that it can form biofilms in the presence of soil clay minerals and iron oxides [47]. This strain has been deposited in the China Centre for Type Culture Collection (CCTCC), and the accession number is CCTCC AB 2018210. The whole genome sequences have been deposited at DDBJ/ENA/
GenBank under accession number QPGT01000000. The $\triangle$ epsG mutant defective for exopolysaccharide (EPS) polymerization was constructed by using homologous recombination as previous described [48]. Escherichia coli strains DH5 $\alpha$ obtained from the State Key Laboratory of Agricultural Microbiology were used for standard DNA manipulations [49]. The kanamycin resistance gene in pDG780 plasmid [49] was inserted into integration sites (epsG) in the genome of $B$. subtilis after inducing electroporation transformation [48]. The primers designed in this study are 1-F:CTAGTCTAGA CGCCCCAAATGGGCAGGC, 1-R:CCGGAATTCCGGTCATGGTCCTTTTCC; 3-F:CCGCTCGAGTTTAT GCACGAGGAGCCG, 3-R:CGGGGTACCGAAGCTGAA AAACTGATC. The relative amounts of bacterial extracellular carbohydrates were estimated by the phenol-sulfuric acid method (see details in the supplementary material) [50]. Planktonic cultures were maintained on Luria-Bertani (LB) medium ( $10 \mathrm{~g}$ of tryptone, $5 \mathrm{~g}$ of yeast extract, and $10 \mathrm{~g}$ of $\mathrm{NaCl}$ per litre of broth) at $37^{\circ} \mathrm{C}$. B. subtilis SBE1 biofilms were cultivated at $37^{\circ} \mathrm{C}$ in minimal salt glycerol glutamate (MSgg) medium [51].

\section{DNase I treatment of biofilm}

The role of extracellular DNA (eDNA) in B. subtilis biofilm formation was investigated by adding DNase I (100 Kunitz units per $\mathrm{mL}$ ) (Sigma-Aldrich, Steinheim, Germany) to an inoculum of $B$. subtilis to degrade the eDNA produced during growth. Briefly, after incubation (see Strains and cultivation conditions above), bacteria were resuspended in MSgg medium [51] and diluted to an $\mathrm{OD}_{600}$ of 0.05 . Next, $200 \mu \mathrm{L}$ of the bacterial suspension was added to each well of a 96-well microtiter plate (Costar, Corning Incorporated, Corning, NY) and 
incubated at $37^{\circ} \mathrm{C}$ without shaking. After 3, 6, 12, 24, 48 , and $72 \mathrm{~h}$, the supernatant from each well was removed, and then the biofilms (pellicles) were washed twice with $1 \mathrm{~mL}$ of sterile $0.9 \% \mathrm{NaCl}$. In addition, $10 \mu \mathrm{L}$ of DNase I (final concentration: 100 Kunitz units per $\mathrm{mL}$ ) was added either at the beginning of the experiment or after the biofilm was established. The biofilm biomass was quantified using crystal violet assays [52]. For each analysis, $1 \% \mathrm{w} / \mathrm{v}$ crystal violet solution $(200 \mu \mathrm{L})$ was added to eight replicate wells, incubated for $10 \mathrm{~min}$, and rinsed twice with $200 \mu \mathrm{L}$ of sterile distilled water. The crystal violet in the residual biofilm was dissolved in $200 \mu \mathrm{L}$ of absolute ethanol. Then, the OD at $595 \mathrm{~nm}$ was measured using a plate reader (PerkinElmer, Waltham, USA).

\section{Atomic force microscopy (AFM)}

The topography of the biofilms with and without DNase I treatment (12 h) was investigated using a MultiMode 8 AFM with a NanoScope V controller (Bruker). The scanning modes used were as follows: 1) ScanAsyst mode using ScanAsyst-Air cantilevers with $0.4 \mathrm{~N} \mathrm{~m}^{-1}$ nominal spring constant (Bruker) and 2) tapping mode using RTESP cantilevers with $40 \mathrm{~N} \mathrm{~m}^{-1}$ nominal spring constant (Bruker) [53]. A scan size of $10 \times 10 \mu \mathrm{m}$ was used. Images were processed and analysed using NanoScope Analysis (Bruker).

\section{Confocal image acquisition and analysis}

The air-liquid interface biofilms were grown in $20 \mathrm{~mm}$ flat bottom cell culture dishes (Costar, Corning Incorporated, Corning, NY). DNase I (100 Kunitz units per mL) was added to the glass chamber during inoculation. For confocal laser scanning microscopy (CLSM) observation, buffer was gently removed from the glass chambers to allow the pellicles to drop onto the glass bottom [53]. The biofilms were stained with a Live/Dead ${ }^{\mathrm{Tm}}$ Bacterial Viability Kit (Bac Light ${ }^{\mathrm{m}}$, Molecular Probes, Invitrogen). The arrangement of the biofilm matrix was determined by direct incorporation of fluorescent labels during synthesis of the exopolysaccharide (EPS) and extracellular DNA (eDNA) matrix, which allowed the examination of the three-dimensional (3D) structure within intact biofilms $[15,54]$. The labelling of EPS and eDNA matrix was performed after biofilms were developed. Extracellular DNA in biofilms was labelled by TOTO-1 nucleic acid stain (cell impermeable, 514/533 nm; Molecular Probes) and PI nucleic acid stain (cell impermeable, 535/ $615 \mathrm{~nm}$; Molecular Probes). The exopolysaccharide matrix was labelled by ConA ( $\alpha$-polysaccharides) (590/ $617 \mathrm{~nm}$; Molecular Probes) [55]. The bacteria in biofilms were labelled by SYTO 9 nucleic acid stain (cell permeable, $485 / 535 \mathrm{~nm}$; Molecular Probes) and SYTO $60 \mathrm{nu}-$ cleic acid stain (cell permeable, 652/678 nm; Molecular Probes). The imaging was performed using an Olympus
FV 1000 monophoton laser scanning microscope (Olympus, Tokyo, Japan) equipped with a $40 \times(0.95$ numerical aperture) objective lens.

The confocal images were analysed by using software to visualize and quantify the bacterial cells, EPS and eDNA within intact biofilms. Imaris 7.4.2 (Bitplane AG, Zurich, Switzerland) was used to rebuild each structural component (bacteria, EPS and eDNA) within the biofilms to visualize the 3D architecture and morphology. Quantitative characterization of each structural component within the $3 \mathrm{D}$ biofilm images was performed as reported previously $[15,56]$. The images were imported to JACoP (Fabrice P. Cordelieres, Institut Curies, Orsay, France), a plugin for ImageJ software [57]. Then, Pearson's coefficient (PC) and the thresholded Mander's coefficients $\mathrm{tM}_{1}$ and $\mathrm{tM}_{2}$ were calculated as described previously $[33,56]$.

\section{DNA purification and preparation of EPS extract}

Genomic DNA of B. subtilis SBE1 (final concentration: $0.003 \mathrm{~mol} / \mathrm{L})$, B. subtilis $3610(0.005 \mathrm{~mol} / \mathrm{L})$, Shewanella oneidensis MR1 $(0.01 \mathrm{~mol} / \mathrm{L})$ and Pseudomonas putida KT2440 $(0.07 \mathrm{~mol} / \mathrm{L})$ was extracted by using Wizard Genomic DNA Purification Kits (Promega). Exopolysaccharides (EPS) were extracted from batch cultures of $B$. subtilis SBE1 as previously described [7]. To remove the DNA, crude EPS was treated with both enzymes, followed by DNase I $\left(100 \mu \mathrm{g} \mathrm{mL} L^{-1}\right)$ for $1 \mathrm{~h}$ at $37^{\circ} \mathrm{C}$ and proteinase $\left.\mathrm{K}(100 \mu \mathrm{g} \mathrm{mL})^{-1}\right)$ for $1 \mathrm{~h}$ at $60^{\circ} \mathrm{C}$.

\section{Isothermal titration calorimetry (ITC)}

Enthalpy changes $(\Delta H)$ of the interaction between DNA and exopolysaccharides (EPS) were determined by isothermal calorimetry using a NANO ITC 2G (TA Instruments, USA). Exopolysaccharides and genomic DNA were dissolved in 0.01 M PBS (pH 7.4). Exopolysaccharides $\left(2.25 \mathrm{mg} \mathrm{mL}^{-1}\right)$ were dispensed into the microcalorimetric cell (volume $1.3 \mathrm{~mL}$ ), and the DNA solution was filled into the syringe compartment (volume $250 \mu \mathrm{L}$ ). DNA was titrated in $10 \mu \mathrm{L}$ portions $(3.14 \mu \mathrm{L}$ for the first injection) into the EPS-containing cell under constant stirring, and the heat of reaction was plotted versus time. All measurements were performed at $25^{\circ} \mathrm{C}$. Data were analysed by using NANOANALYZE software [58].

\section{Statistical analysis}

Data were basically described by means and respective standard deviations (SD). $P$-values were acquired using analysis of variance (ANOVA) followed by Tukey's multiple comparisons test to evaluate statistical significance using SPSS 17.0 software. Differences were regarded as statistically significant when $p<0.05$. 


\section{Supplementary information}

Supplementary information accompanies this paper at https://doi.org/10. 1186/s12866-020-01789-5.

Additional file 1 Supplementary Information contains (1) additional details on the materials and methods; (2) a figure of structural arrangement of bacteria during biofilm development of $B$. subtilis SBE1 and its exopolysaccharide (EPS) mutant ( $\triangle e p s G)$; (3) a figure of total carbohydrate content of EPS produced by $B$. subtilis SBE1 and its

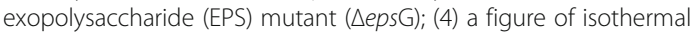
titration calorimetry measurement to determine the interaction of exopolysaccharide (EPS) and genomic DNA

\section{Abbreviations}

eDNA: Extracellular DNA; EPS: Exopolysaccharide; LTA: Lipoteichoic acid; LPS: Lipid polysaccharide; 3D: Three-dimensional; HGT: Horizontal gene transfer; CCTCC: China Centre for Type Culture Collection; LB: Luria-Berta; MSgg: Minimal salt glycerol glutamate; CLSM: Confocal laser scanning microscopy; AFM: Atomic force microscopy; ITC: Isothermal titration calorimetry; $\Delta H$ : Enthalpy changes; SD: Standard deviations; ANOVA: Analysis of variance; PC: Pearson's coefficient; M1thr: Thresholded Mander's $\mathrm{tM}_{1}$; M2thr: Thresholded Mander's $\mathrm{tM}_{2}$

\section{Acknowledgments}

We thank Zhe Hu for the help in technical matters of microscope technology.

\section{Authors' contributions}

N. P. conceived the experimental strategy, conducted experiments, acquired data, analyzed data and wrote the manuscript. P. C. acquired funding, conceived the experimental strategy, wrote the manuscript. M. M wrote the manuscript. Y. C. W and C. H. G analyzed data. Q. Y. H wrote the manuscript. All authors have read and approved the manuscript.

\section{Funding}

This work was supported by the National Natural Science Foundation of China (41877029, 41961130383), Royal Society-Newton Advanced Fellowship (NAF\R1\191017), the National Key Research Program of China (2016YFD0800206) and Wuhan Science and Technology Bureau (2019020701011469). The funders had no role in the study design, data collection and analysis, decision to publish, or preparation of the manuscript.

\section{Availability of data and materials}

Bacillus subtilis SBE1 used in this study has been deposited in the China Centre for Type Culture Collection (CCTCC), and the accession number is CCTCC AB 2018210. The whole genome sequences of this strian have been deposited at DDBJ/ENA/GenBank under accession number QPGT01000000.The dataset supporting the conclusions of this article is included within the article (and its Additional files S1-3).

\section{Ethics approval and consent to participate}

Not applicable.

\section{Consent for publication}

Not applicable.

\section{Competing interests}

The authors declare that they have no competing interests.

\section{Author details}

${ }^{1}$ State Key Laboratory of Agricultural Microbiology, College of Resources of Environment, Huazhong Agricultural University, Wuhan 430070, China. ${ }^{2}$ Bren School of Environmental Science and Management and Earth Research Institute, University of California, Santa Barbara, California 93106, USA.

Received: 28 November 2019 Accepted: 16 April 2020

Published online: 14 May 2020

\section{References}

1. Ghannoum M, OToole GA. Microbial Biofilms. Washington, DC: ASM Press; 2004
2. Stewart PS, Franklin MJ. Physiological heterogeneity in biofilms. Nat Rev Microbiol. 2008;6:199-210.

3. López D, Kolter R. Functional microdomains in bacterial membranes. Genes Dev. 2010;24:1893-902.

4. Branda SS, Vik Å, Friedman Land Kolter R. Biofilms: the matrix revisited. Trends Microbiol. 2005;13(1):0-26.

5. Flemming H-C, Wingender J. The biofilm matrix. Nat Rev Microbiol. 2010;8:623-33.

6. Whitchurch CB, Tolker-Nielsen T, Ragas PC, Mattick JS. Extracellular DNA required for bacterial biofilm formation. Science. 2002;295:1487.

7. Dominiak DM, Nielsen JL, Nielsen PH. Extracellular DNA is abundant and important for microcolony strength in mixed microbial biofilms. Environ Microbiol. 2011;13:710-21.

8. Finkel SE, Kolter R. DNA as a nutrient: novel role for bacterial competence gene homologs. J Bacteriol. 2001;183:6288-93.

9. Molin S, Tolker-Nielsen T. Gene transfer occurs with enhanced efficiency in biofilms and induces enhanced stabilization of the biofilm structure. Curr Opin Biotechnol. 2003;14:255-61.

10. Qin $Z$, Ou $Y$, Yang $L$, et al. Role of autolysin-mediated DNA release in biofilm formation of Staphylococcus epidermidis. Microbiology. 2007;153:2083-92.

11. Hall-stoodley L, Nistico L, Sambanthamoorthy K, et al. Characterization of biofilm matrix, degradation by DNase treatment and evidence of capsule downregulation in Streptococcus pneumoniae clinical isolates. BMC Microbiol. 2008:8:173.

12. Seper A, Fengler VHI, Roier $\mathrm{S}$, et al. Extracellular nucleases and extracellular DNA play important roles in Vibrio cholerae biofilm formation. Mol Microbiol. 2011;82:1015-37.

13. Ma L, Conover M, Lu H, Parsek MR, Bayles K, Wozniak DJ. Assembly and development of the Pseudomonas aeruginosa biofilm matrix. PLoS Pathog. 2009;5:e1000354.

14. Sutherland IW. In: Kamerling JP, editor. Comprehensive Glycoscience Vol. 2. Doordrecht: Elsevier; 2007. p. 521-58.

15. Xiao J, Klein MI, Falsetta ML, et al. The exopolysaccharide matrix modulates the interaction between 3D architecture and virulence of a mixed-species oral biofilm. PLoS Pathog. 2012;8:e1002623.

16. Liao S, Klein MI, Heim KP, et al. Wen. Streptococcus mutans extracellular DNA is upregulated during growth in biofilms, actively released via membrane vesicles, and influenced by components of the protein secretion machinery. J Bacteriol. 2014;196:2355-66.

17. Wang S, Liu X, Zhang $L$, et al. The exopolysaccharide Psl-eDNA interaction enables the formation of a biofilm skeleton in Pseudomonas aeruginosa. Environ Microbiol Rep. 2015;7(2):330-40.

18. Bais HP, Fall R, Vivanco JM. Biocontrol of Bacillus subtilis against infection of Arabidopsis roots by Pseudomonas syringae is facilitated by biofilm formation and surfactin production. Plant Physio. 2004;134(1):307-19.

19. Stein T, Dusterhus S, Stroh A, Entian KD. Subtilosin production by two Bacillus subtilis subspecies and variance of the sbo-alb cluster. Appl Environ Microbiol. 2004:70:2349-53.

20. Butcher RA, Schroeder FC, Fischbach MA, et al. The identification of bacillaene, the product of the PksX megacomplex in Bacillus subtilis. Proc Natl Acad Sci U S A. 2007;104:1506-9.

21. Nagorska K, Bikowski M, Obuchowskji M. Multicellular behaviour and production of a wide variety of toxic substances support usage of Bacillus subtilis as a powerful biocontrol agent. Acta Biochim Pol. 2007;54:495-508.

22. Ongena M, Jourdan E, Adam A, et al. Surfactin and fengycin lipopeptides of Bacillus subtilis as elicitors of induced systemic resistance in plants. Environ Microbiol. 2007;9:1084-90.

23. Ongena M, Jacques P. Bacillus lipopeptides: versatile weapons for plant disease biocontrol. Trends Microbiol. 2008;16:115-25.

24. Chen Y, Cao S, Chai Y, et al. A Bacillus subtilis sensor kinase involved in triggering biofilm formation on the roots of tomato plants. Mol Microbiol. 2012;85:418-30.

25. Chen Y, Yan F, Chai Y, et al. Biocontrol of tomato wilt disease by Bacillus subtilis isolates from natural environments depends on conserved genes mediating biofilm formation. Environ Microbiol. 2013;15:848-64.

26. Beauregard PB, Chai Y, Vlamakis H, Losick R, Kolter R. Bacillus subtilis biofilm induction by plant polysaccharides. Proc Natl Acad Sci U S A. 2013;110:E1621-30.

27. Branda SS, Chu F, Kearns DB, Losick R, Kolter R. A major protein component of the Bacillus subtilis biofilm matrix. Mol Microbiol. 2006;59:1229-38.

28. Sinha RP, lyer VN. Competence for genetic transformation and the release of DNA from Bacillus subtilis. Biochimica et Biophysica Acta (BBA)-Nucleic Acids and Protein. Synthesis. 1971;232(1):61-71. 
29. López D, Vlamakis H, Losick R, Kolter R. Cannibalism enhances biofilm development in Bacillus subtilis. Mol Microbiol. 2009;74:609-18.

30. Crabb WD, Streips UN, Doyle RJ. Selective enrichment for genetic markers in DNA released by competent cultures of Bacillus subtilis. Mol Gen Genet. 1977; 155:179-83.

31. Ibáñez de Aldecoa AL, Zafra O, González-Pastor JE. Mechanisms and regulation of extracellular DNA release and its biological roles in microbial communities. Front Microbiol. 2017;8:1390.

32. Zafra O, Lamprecht-Grandío M, González de Figueras C, González-Pastor JE. Extracellular DNA release by undomesticated Bacillus subtilis is regulated by early competence. PLoS One. 2012;7:e48716.

33. Harmsen M, Lappann M, Knøchel S, Molin S. Role of extracellular DNA during biofilm formation by Listeria monocytogenes. Appl Environ Microbiol. 2010;76:2271-9.

34. Hobley L, Harkins C, Macphee CE, Stanleywall NR. Giving structure to the biofilm matrix: an overview of individual strategies and emerging common themes. FEMS Microbiol Rev. 2015;39:649-69.

35. Branda SS, González-Pastor JE, Ben-Yehuda S, Losick R, Kolter R. Fruiting body formation in Bacillus subtilis. Proc Natl Acad Sci U S A. 2001:98:11621-6.

36. Kearns DB, Chu F, Branda SS, Kolter R, Losick R. A master regulator for biofilm formation by Bacillus subtilis. Mol Microbiol. 2005;55:739-49.

37. Gerwig J, Kiley TB, Gunka K, Stanley-Wall N, Stulke J. The protein tyrosine kinases EpsB and PtkA differentially affect biofilm formation in Bacillus subtilis. Microbiology. 2014;160:682-91.

38. Guttenplan SB, Blair KM, Kearns DB. The EpsE flagellar clutch is bifunctional and synergizes with EPS biosynthesis to promote Bacillus subtilis biofilm formation. PLoS Genet. 2010;6:e1001243.

39. Terra R, Stanley-Wall NR, Cao G, Lazazzera BA. Identification of Bacillus subtilis sipW as a bifunctional signal peptidase that controls surface-adhered biofilm formation. J Bacteriol. 2012;194:2781-90.

40. Yang L, Hu Y, Liu Y, Zhang J, Ulstrup J, Molin S. Distinct roles of extracellular polymeric substances in Pseudomonas aeruginosa biofilm development. Environ Microbiol. 2011;13:1705-17.

41. Castillo Pedraza MC, Novais TF, Faustoferri RC, et al. Klein. Extracellular DNA and lipoteichoic acids interact with exopolysaccharides in the extracellular matrix of Streptococcus mutans biofilms. Biofouling. 2017:33:722-40.

42. Gries A, Prassl R, Fukuoka S, et al. Biophysical analysis of the interaction of the serum protein human beta2GPI with bacterial lipopolysaccharide. FEBS Open Bio. 2014:4:432-40

43. Tawfik DS. Accuracy-rate tradeoffs: how do enzymes meet demands of selectivity and catalytic efficiency? Curr Opin Chem Biol. 2014;21:73-80.

44. Grundmann GL. Spatial scales of soil bacterial diversity - the size of a clone. FEMS Microbiol Ecol. 2004;48:119-27.

45. Nunan N, Wu KJ, Young IM, Crawford JW, Ritz K. Spatial distribution of bacterial communities and their relationships with the micro-architecture of soil. FEMS Microbiol Ecol. 2003;44:203-15.

46. Burmølle $M$, Thomsen TR, Fazli $M$, et al. Biofilms in chronic infections - a matter of opportunity monospecies biofilms in multispecies infections. FEMS Immunol Med Microbiol. 2010;59:324-36.

47. Ma W, Peng D, Walker SL, et al. Bacillus subtilis biofilm development in the presence of soil clay minerals and iron oxides. NPJ Biofilms Microbiomes. 2017:3:4

48. Dubnau D. Genetic exchange and homologous recombination. Bacillus subtilis and other gram-positive bacteria. Am Soc Microbiol. 1993. p. 555-84.

49. Cue D, Lam H, Dillingham RL, et al. Genetic manipulation of Bacillus methanolicus, a gram-positive, thermotolerant methylotroph. Appl Environ Microbiol. 1997;63:1406-20.

50. Wecke $\mathrm{T}$, Bauer $\mathrm{T}$, Harth $\mathrm{H}$, et al. The rhamnolipid stress response of Bacillus subtilis. FEMS Microbiol Lett. 2011;323:113-23.

51. Brimacombe CA, Stevens A, Jun D, Mercer R, Lang AS, Beatty JT. Quorumsensing regulation of a capsular polysaccharide receptor for the Rhodobacter capsulatus gene transfer agent (RcGTA). Mol Microbiol. 2013;87:802-17.

52. Coffey BM, Anderson GG. Biofilm formation in the 96-well microtiter plate. In: Pseudomonas Methods and Protocols (pp. 631-641). New York: Humana Press; 2014.

53. Gloag ES, Turnbull L, Huang A, et al. Self-organization of bacterial biofilms is facilitated by extracellular DNA. Proc Natl Acad Sci. 2013;110(28):11541-6.

54. Okshevsky M, Meyer RL. Evaluation of fluorescent stains for visualizing extracellular DNA in biofilms. J Microbiol Meth. 2014;105:102-4.

55. Yu GH, Tang Z, Xu YC, Shen QR. Multiple fluorescence labeling and two dimensional FTIR- ${ }^{13} \mathrm{C}$ NMR heterospectral correlation spectroscopy to characterize extracellular polymeric substances in biofilms produced during composting. Environ Sci Technol. 2011;45:9224-31.
56. Xiao J, Koo H. Structural organization and dynamics of exopolysaccharide matrix and microcolonies formation by Streptococcus mutans in biofilms. Appl Microbiol. 2010;108:2103-13.

57. Rasband WS. Image J. Bethesda, MD, U.S.A: US National Institutes of Health; 1997-2006.

58. Ni B, Huang Z, Fan Z, Jiang CY, Liu SJ. Comamonas testosteroni uses a chemoreceptor for tricarboxylic acid cycle intermediates to trigger chemotactic responses towards aromatic compounds. Mol Microbiol. 2013; 90:813-23.

\section{Publisher's Note}

Springer Nature remains neutral with regard to jurisdictional claims in published maps and institutional affiliations.
Ready to submit your research? Choose BMC and benefit from:

- fast, convenient online submission

- thorough peer review by experienced researchers in your field

- rapid publication on acceptance

- support for research data, including large and complex data types

- gold Open Access which fosters wider collaboration and increased citations

- maximum visibility for your research: over $100 \mathrm{M}$ website views per year

At $\mathrm{BMC}$, research is always in progress.

Learn more biomedcentral.com/submissions 